\title{
APORTACION AL ESTUDIO DE LOS HONGOS DE ANDALUCIA I. ESPECIES NUEVAS O INTERESANTES DE LA PROVINCIA DE GRANADA
}

\author{
A. ORTEGA* y F. D. CALONGE**
}

\section{RESUMEN:}

Se describen Hebeloma eburneum Malenç. como nueva cita para Europa; Cortinarius bolophaeus J. Lange, Helvella pezizoides Afz. ex Fr., Ptychoverpa bohemica (Kromb.) Boud. y Russula straminea Malenç., como nuevas para España; Hebeloma pallidum Malenç., nuevo para España peninsular y finalmente se citan Geopora foliacea (Schiff. ex Boud.) Torre, Scutellinia umbrorum (Fr.) Lamb., Cortinarius berculeus Malenç., C. ionocblorus Maire, Hebeloma edurum Metrod, Myriostoma coliforme (Dicks. ex Pers.) Corda, Mycenastrum corium (Guers. ex Lamb. et DC.) Desv. y Scleroderma bovista Fr., como nuevas para Andalucía.

\section{SUMMARY:}

Fourteen species of fungi collected within the province of Granada, Spain, are described or mentioned here, which can be grouped as follows: Hebeloma eburneum Malenç., as new record to Europe; Cortinarius holophaeus J. Lange, Helvella pezizoides Afz. ex Fr., Ptychoverpa bobemica (Kromb.) Boud. and Russula straminea Malenç., as new to Spain; Hebeloma paIlidum Malenç., as new to Peninsular Spain, and finally Geopora foliacea (Schff. ex Boud.) Torre, Scutellinia umbrorum (Fr.) Lamb., Cortinarius berculeus Malenç., C. ionochlorus Maire, Hebeloma edurum Metrod, Myriostoma coliforme (Dicks. ex Pers.) Corda, Mycenastrum corium (Guers. ex Lamb. et DC.) Desv., and Scleroderma bovista Fr., as new records to Andalusia

\section{INTRODUCCION}

Aunque no muy numerosos, si se pueden encontrar en la literatura algunos artículos en los que se mencionan o describen hongos, procedentes de la región andaluza. Entre los más importantes, y siguiendo un orden cronológico, podríamos citar los de Colmeiro (1868), Amo y Mora (1870), Hernández 
Pacheco (1901), Díez Tortosa (1909), González Fragoso (1883, 1917), Lázaro e Ibiza (1920), Maire (1937, 1943), Muñoz Medina \& Serrano Sánchez(1945), Esteve \& Varo (1967) Malencon (1968), Bertault (1974), Moreno(1976) y Esteve, Varo, Ortega \& Ramírez (1978).

El objeto del presente trabajo es el de iniciar un estudio, lo más profundo posible, sobre la flora micológica de la provincia de Granada, comenzandopor describir aquellas encontradas recientemente, que constituyen novedad para el catálogo micológico español, y seguidamente dar paso a la cita de aquellas otras que son nuevas para Andalucía, con la indicación de las notas ecológicas correspondientes a cada una de ellas.

\section{DESCRIPCION DE ESPECIES}

\section{Hebelona eburneum Malenç.}

Macroscopía. Todo el material recolectado coincide bastante bien con la descripción dada para esta especie por Malençon y Bertault (1970). No obstante, teniendo en cuenta que se trata de una especie recientemente descrita, vamos a destacar algunas de sus características diferenciales.

El sombrero puede llegar a medir hasta $10 \mathrm{~cm}$., de diámetro, aplanándose al llegar a la madurez y con los bordes fuertemente lobulados a veces. Cutícula viscosa en tiempo húmedo, de tono crema pálido, algo más oscuro en el centro. Láminas anchas, apretadas, casi libres, de un color crema tendiendo al rosado. Pie cilíndrico, de 5-8 x 1-2 cm., macizo, robusto, con la cutícula algo más pálida que las láminas y recubierta de pequeñas escamas de tono ligeramente más oscuro. Carne blanquecina, compacta en el sombrero y algo fibrosa en el pie, con sabor dulzaino. (Fig. 1).

Microscopía. Las esporas son de forma de almendra, con la superficie suavemente verrugosa, de tono amarillo ocráceo y con unas dimensiones de 9, 5-13 x 6-7,2 u. (Figura 1).

Habitat. Siempre crece bajo cedros, existiendo, al parecer, una estrecha relación simbiótica entre el hongo y el cedro.

Localidad. Se ha encontrado en La Alfaguara, Granada (17-XII-77).

Leg. G. Malençon, X Llimona y A. Ortega.

Observaciones. Hasta el momento, que nosotros sepamos, sólo se ha encontrado en Marruecos, en la zona del Atlas Medio. Por lo tanto esta nueva localidad representa la primera para España y Europa a la vez; siendo probable que este hongo esté presente también en otras zonas del área mediterránea.

\section{Cortinarius holophaeus J. Lange non Henry}

Macroscopía. Sombrero plano-convexo de 3-4,5 cm., de diámetro de color pardo oscuro, aclarándose en ciertas zonas por la presencia de fibrillas radiales pálidas. Pie de 6-11 x 0,5-1 $\mathrm{cm}$., cilíndrico, algo ensanchado en la base frágil, del mismo color que el sombrero y también cubierto de unas fibrillas muy delicadas. Láminas de color pardo oscuro, ventrudas y 


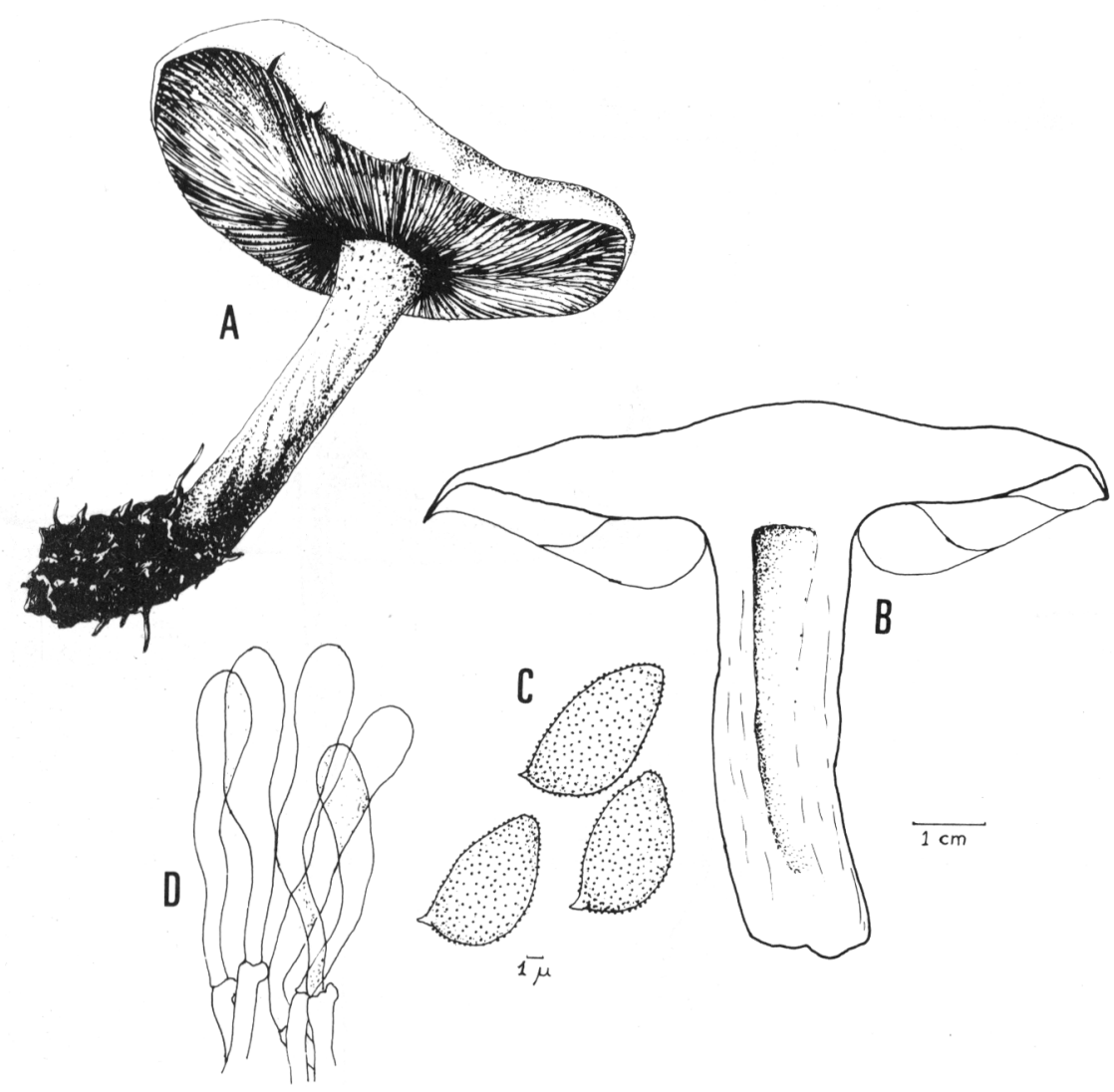

Fig. 1.-Hebeloma eburneum Mal. A Carpóforo; B Corte longitudinal del carpóforo; CEsporas; D Pelos de la arista.

numerosas. La carne es de color blanco amarillento, delgada en espesor y de sabor dulzaino casi imperceptible. (Fig. 2).

Microscopía. Esporas de ovales a elípticas de 9,5-10,5 x 5,5-6,5 u., con la superficie bastante verrugosa, y una tonalidad marrón ferruginosa. (Fig. 2).

Habitat. La encontramos creciendo en un bosque de Quercus pyrenaica en suelo rico en mantillo y hojas semidescompuestas de este árbol.

Localidad. Hostal del Duque, Sierra Nevada, Granada a 1.300 m. alt., Leg. A. Ortega(1210-77), G. Malençon, X. Llimona y A. Ortega (18-10-77), J. Varo y A. Ortega (8-11-77).

Observaciones. Es nueva cita para España. 


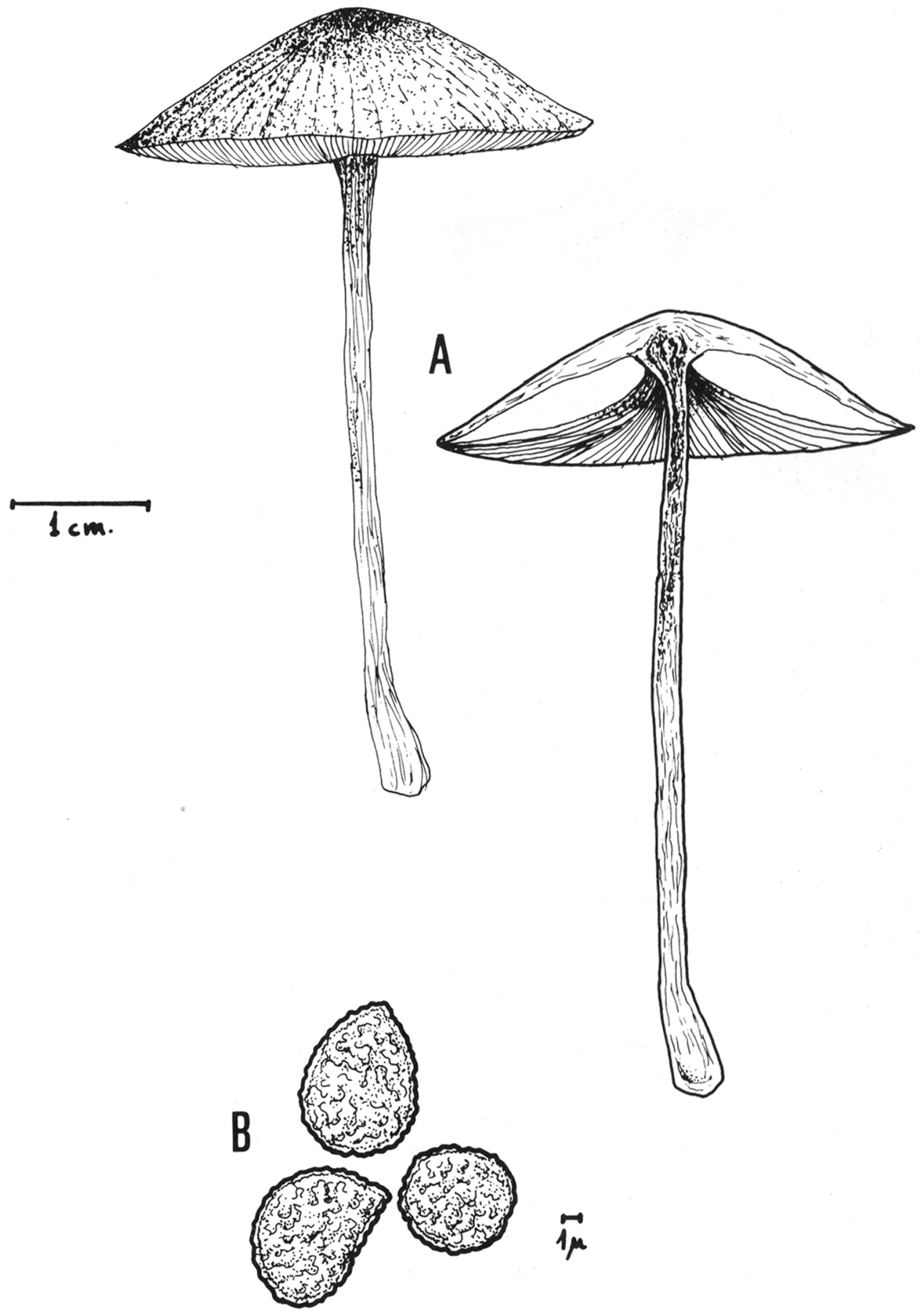

Fig. 2.-Cortinarius holophaeus J. Lange; A Carpóforo, B Esporas. 
Helvella pezizoides Afz. ex Fr.

Macroscopía. Sombrero de 1-2 cm., de diámetro, algo ciatiforme, finalmente con los bordes muy ondulados y a veces tendiendo a formar como sillas de montar. La cara himenial es marrón oscura, casi negra. La parte externa es algo más clara y suavemente tomentosa, apreciándose este carácter a simple vista. El pie es delgado de 1-3 centímetros de longitud con un grosor de 2-4 mm., del mismo color que la cara externa del sombrero y también con el mismo tipo de tomento.

Microscopía. Ascos cilíndricos de 280-330 x 13-16 u., parafisos de igual longitud y de 3-6 u., de anchura, ligeramente ensanchados en el ápice, con tonalidad marrón en su pared. Esporas ovales a elípticas con tendencia a ser cilíndricas, lisas, midiéndo de 15-18 x 9-11 u. Interiormente las esporas presentan una gran gota lipídica que ocupa casi la totalidad del contenido.

Habitat. Se encontró viviendo bajo castaños en un suelo húmico.

Localidad. Barranco de San Juan, Sierra Nevada, Granada, a 1.330 m. alt. Leg. A. Ortega (7-10-77).

Observaciones. Es una nueva cita para España. Su distribución en el mundo es bastante amplia; habiendo sido citada hasta ahora en Suecia, Finlandia, Dinamarca, Inglaterra, Alemania, Holanda, Francia, Rusia, Italia, Portugal, Pakistan y U.S.A. (Dissing, 1966).

Desde el punto de vista botánico esta especie presenta un gran parecido con la $\mathrm{H}$. atra Holm., y H. ephippium Lév. De la $H$. atra se diferencia porque esta presenta la parte externa del sombrero de color gris y sin tomento y de la $H$. ephippium en que esta presenta un himenio de color gris, aunque exteriormente también es tomentosa (Dissing, 1966).

Ptychoverpa bohemica (Kromb.) Boud.

Verpa bispora Sorok. V. gigaspora Cke.

Macroscopía. Sombrero de 2,5-4 x 2,5-3,5 cm., cónico acampanado con costillas longitudinales sinuosas que recuerdan una masa cerebral. En esta cara, que presenta un color marrón castaño, es donde se asienta el himenio. (Fig. 3).

La cara interior es lisa y muestra un color crema pálido. El pie es cilíndrico, de 10-12x 1-2 cm; exteriormente presenta un color blanquecino, con zonas anulares algodonosas suaves que le dan un aspecto cebrado típico. Interiormente es hueco. La carne es blanda, de color crema pálida y carente de sabor y olor, aparte del normalolor de arcilla mojada que presentan la mayoría de los hongos.

Microscopía. Los ascos, normalmente bispóricos, miden de 220-240 x 18-24 u., y las esporas son típicas por el enorme tamaño que presentan, de 65-75 x 18-20 u. de forma elíptica, estrechas y algo curvadas, con la superficie lisa. (Fig. 3).

Habitat. Viviendo en el suelo de una alameda entre las hojas caídas de Populus nigra.

Localidad. Río Maitena, a 1.150 m., alt., Sierra Nevada, Granada Leg. a. Ortega (16-4-78).

Observaciones. Primera cita para España. Se comporta, en cuanto a su distribución, como centro europeo de montaña, siendo este hallazgo español muy importante por lo que significa para el conocimiento de su área de dispersión. 


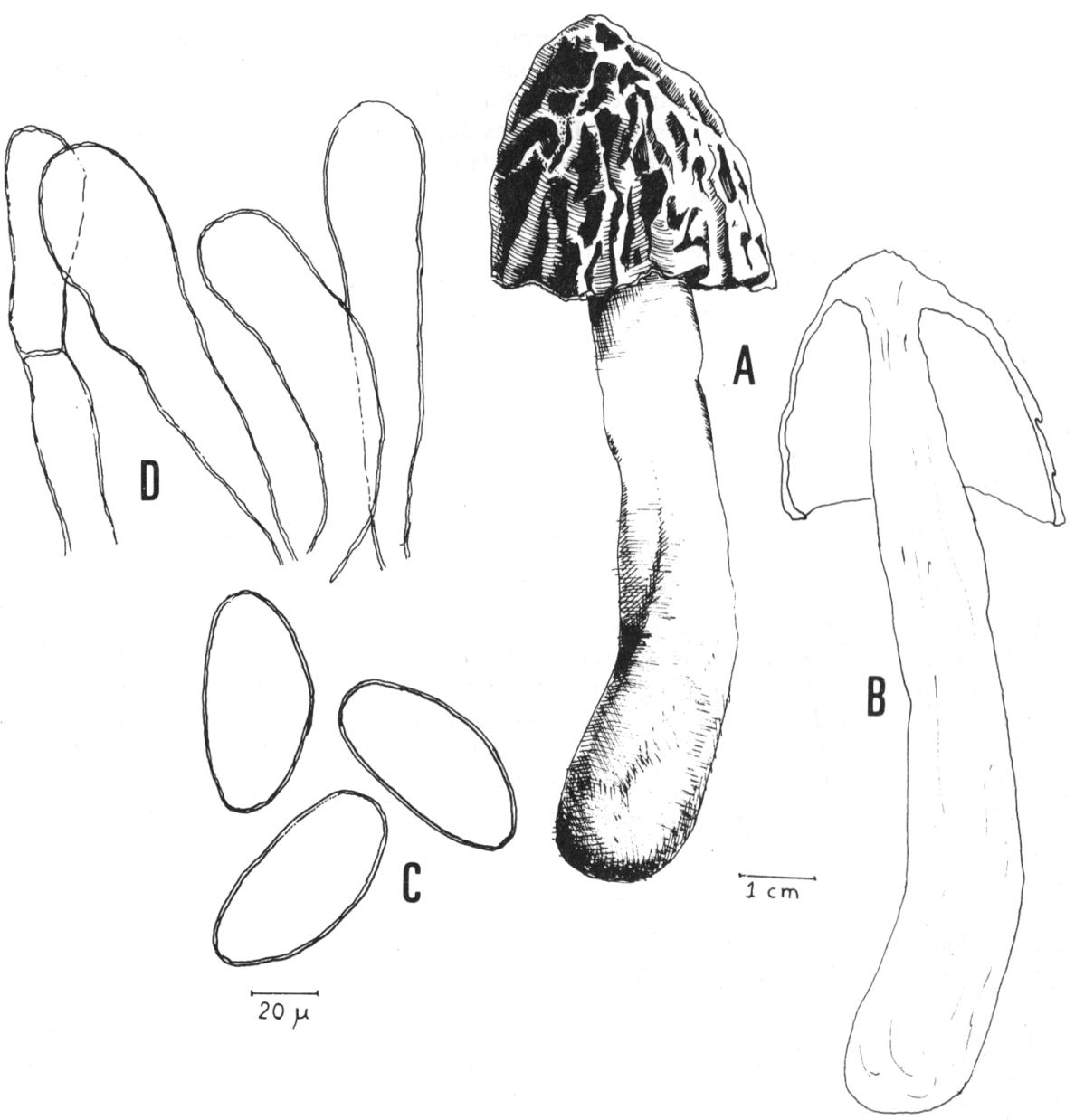

Fig. 3.-Ptychoverpa bohemica (Kromb.) Boud. A Carpóforo; B Corte longitudinal del carpóforo; C Esporas; D Ascas y parafisos.

\section{Russula straminea Malenç.}

Macroscopía. Sombrero de $8-12 \mathrm{~cm}$., de diámetro, plano al principio y embudado después, de color crema amarillento y con estrias radiales. La cutícula es viscosa en tiempo húmedo y fácil de separar. Las láminas son anchas, numerosas, tendiendo a decurrentes, blancas al principio y crema ocráceas al final. El pie es cilindráceo, atenuándose hacia la base, de 5-7 x 2-3 cm., robusto, blanco, que mas tarde pasa a crema sucio con la edad. La carne es esponjosa, blanquecina, de sabor dulzaino e inodora. Esporada color ocre. (Fig. 4).

Microscopía. Esporas grandes, de 10-13 x 9-10 u., de forma oval a globosa, cubiertas con abundantes espinas cónicas que miden de 0,6-0,9 u. En la cutícula se pueden observar 
abundantes dermatocistidios, obtusos en el ápice, con septos frecuentes y con un grosor de 9-12 u. (Fig. 4).

Habitat. Crece en encinares de Quercus rotundifolia mezclados con Pinus pinaster.

Localidad. Camino del Campamento de la Alfaguara, Sierra de Alfacar, Granada. Leg. G. Malençon, X. Llimona y A. Ortega (18-10-77).

Observaciones. Especie nueva para España, que ha sido citada hasta el momento en Marruecos (Malençon y Bertault, 1970), y en Suiza (Huissjman).

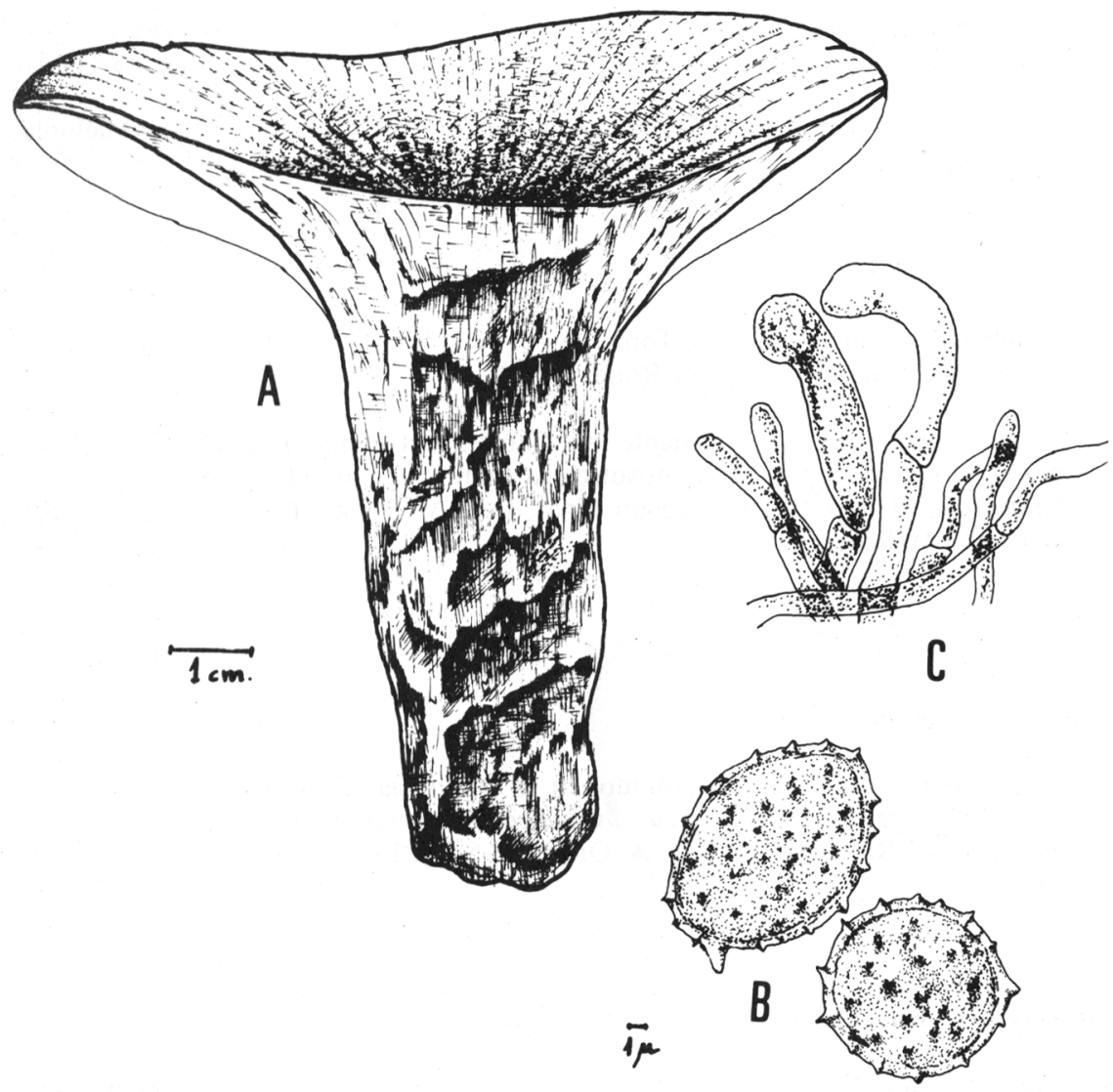

Fig. 4.-Kussula straminea Mal. A Corte longitudinal del carpóforo, B Esporas, C Cutícula: Dermatocistidios 


\section{Hebeloma pallidum Malenç.}

Macroscopía. Sombrero de 3-5 cm., de diámetro, al principio semiesférico después planoconvexo conservando siempre un mamelón central. Cutícula de color blanco crema, viscosa en tiempo húmedo. Margen redondeado, a veces con lóbulos bien marcados. Láminas anchas, poco apretadas, desiguales, sinuadas y de un tono crema ocraceo. Esporada de color amarillo ocráceo. Pie cilíndrico de $3-5 \times 0,6-0,8 \mathrm{~cm}$., generalmente curvado, finamente estriado, de igual color que el sombrero y hueco en el interior. Carne blanquecina, esponjosa, algo fibrosa en el pie, inodora e insípida.

Microscopía. Esporas ovales de 9,5-10 x 5,3-5,7 u., con membrana lisa o suavemente verrugosa.

Habitat. Se ha recolectado viviendo en bosque mixto de Pinus pinaster, Quercus rotundifolia y $Q$. pyrenaica.

Localidad. Sierra de Alfacar, Granada; Leg. G. Malençon, X. Llimona y A. Ortega (17-1077); Hostal del Duque, 1.300 m. alt., Sierra Nevada, Leg. J. Varo y A. Ortega (8-11-77).

Observaciones. Nueva para España peninsular. Anteriormente se había citado en Mallorca creciendo bajo Quercus ilex y Pinus halepensis (Malençon y Bertault, 1972).

Geopora foliacea (Schff. ex Boud.) Torre

Sepultaria foliacea (Schff. ex Boud.) Boud.

Obs.-Especie encontrada anteriormente en Cataluña (Unamuno, 1941; .Rivas-Martínez y Losa Quintana, 1969); nosotros la hemos encontrado en Sierra Alhama, Granada, viviendo en suelo de un pinar. Leg. A. Ortega (6-2-78). Es una nueva cita para Andalucía.

\section{Scutellinia umbrorum (Fr.) Lamb.}

Obs.-Anteriormente se había encontrado en la provincia de Segovia (Torre, 1974), en suelo de bosque de Quercus pyrenaica. El material granadino procede del Barranco del Río Bermejo. Leg. A. Ortega (8-5-78). Es una nueva cita para Andalucía.

\section{Cortinarius herculeus Malenç.}

Obs.-Citada ya para Cataluña (Malençon y Bertault, 1971), nosotros la hemos encontrado en la provincia de Granada en la localidad de Alfaguara, en el lugar conocido como El Jardín. Leg. A. Ortega (26-10-77). Es nueva cita para Andalucía. Lo más típico de esta especie, aparte de su robustez, pues alcanza los 17 centímetros de diámetro en el sombrero, es su dependencia del cedro; siempre aparece creciendo bajo cedros, como norma general. 


\section{Cortinarius ionochlorus Maire}

Obs.-Se ha citado anteriormente en Cataluña (Malençon y Bertault, 1971). Nosotros la hemos recolectado en Fuente Fría, Alfaguara, leg. A. Ortega (4-11-77), creciendo bajo Quercus rotundifolia. Es nueva, por tanto, para Andalucía. (Fig. 5).

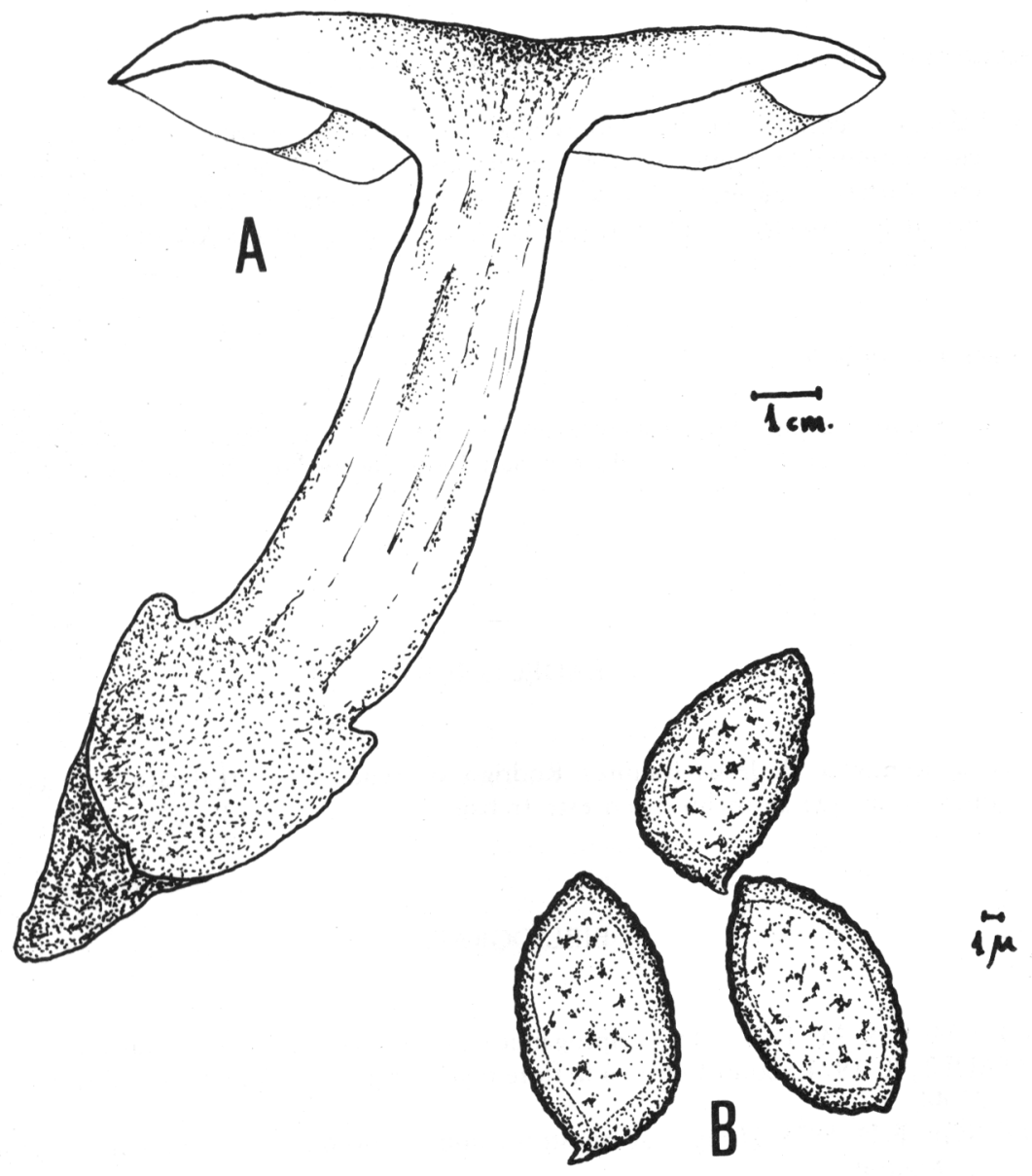

Fig. 5.-Cortinarius ionochlorus Mre. A Carpóforo, B Esporas.

\section{Hebeloma edurum Metrod}

Obs.-Citado anteriormente en Cataluña y Baleares (Malençon y Bertault, 1971, 1972, 1976), en Granada se ha encontrado en el Camino del Campamento de la Alfaguara. Leg. G. Malençon, X. Llimona y A. Ortega (18-20-77), Sierra de Alfacar (Oct. Nov. 1977), leg. A. Ortega. Nueva cita para Andalucía. 
Myriostoma coliforme (Dicks. ex Pers.) Corda

Obs.-Se ha encontrado en la Vereda de la Estrella, $1.300 \mathrm{~m}$. alt., Sierra Nevada, bajo Quercus pyrenaica. Leg. A. Ortega (1-11-78). Hasta ahora sólo se había encontrado en la provincia de Madrid (Calonge, 1976), con lo que se amplía significativamente el área de dispersión de esta especie. Se identifica a primera vista por su endoperíodo con múltiples ostiolos.

Mycenastrum corium (Guers. ex Lamb. \& DC.) Desv.

Obs.-Fácil de reconocer por su peridio blanco y coriáceo, y sobre todo por su capilicio fuertemente espinoso, visto al microscópio. Anteriormente se había citado en Asturias, Madrid, País Vasco y Canarias (Calonge y Demoulin, 1975). Nosotros lo hemos encontrado en Granada en la localidad de Alfaguara el día 17-677, leg. A. Ortega y F. Valle.

\section{Scleroderma bovista Fr.}

Obs.-Solamente se ha citado una vez en Cataluña (Maire, 1933). Nosotros la hemos encontrado en la localidad de Río San Juan, Sierra Nevada. Leg. A. Ortega (2210-77).

\section{AGRADECIMIENTOS}

Agradecemos a D. Jesús Ramírez Rodrígo su ayuda en la realización de las figuras esquemáticas que se incluyen en este trabajo.

\section{BIBLIOGRAFIA}

AMO y MORA, M. 1870. Flora criptogámica de España y Portugal. Granada.

BERTAULT, R. 1974. Contribution à la flore mycologique de l'Andalousie. Collect. Bot. 9 : 25-44.

CALONGE, F. D. 1976. Hongos de nuestros campos y bosques Pub. Ministerio de Agricultura. Madrid.

CALONGE, F.D. \& DEMOULIN, J. 1975. Les Gasteromycetes d'Espagne. Bull. Soc. Mycol. France, 91: 247-292.

COLMEIRO, M. 1868. Enumeración de las criptógamas de España y Portugal. Revista para el Progreso de las Ciencias. T. 16-68. Madrid.

DIEZ TORTOSA, J.L. 1909. Datos para la flora micológica de la región meridional de España. Bol. Soc. Esp. Hist. Nat., 9: 95-100.

DISSING, H. 1966. The genus Helvella in Europe, with special emphasis on the species found in Norden. Dansk Botanisk Arkiv, 25 (1): 172 pp.

ESTEVE, F. \& VARO, J. 1967. Colus hirudinosus Cav. \& Sech., género nuevo para la flora micológica meridional hispánica, denunciado en Adamuz (Córdoba). Ars Pharm., 8: 11-12. 
ESTEVE, F.; VARO, J.; ORTEGA, A. \& RAMIREZ, J. 1978. Algunos hongos de interés bromatológico y farmacéutico de la provincia de Granada. Ars Pharm., 19: 125-150.

GONZALEZ FRAGOSO, R. 1883. Flora de la provincia de Sevilla. Criptógamas. An. Soc. Esp. Hist. Nat., 12: 1-28.

GONZALEZ FRAGOSO, R. 1917. Hongos de la provincia de Málaga. Bol. Soc. Esp. Hist. Nat., 17: 299-311.

HERNANDEZ PACHECO, E. 1901. Datos para la flora micológica de los alrededores de Córdoba. Bol. Soc. Esp. Hist. Nat., 1: 131-133.

LAZARO e IBIZA, B. 1920. Compendio de la flora española. Madrid.

MAIRE, R.; CODINA, J. \& FONT QUER, P. 1933. Fungi catalaunici. Contribution à l'etude de la flore mycologique de la Catalogne. Treballs Museum Cienc. Nat. Barcelona, 15: 1-120.

MAIRE, R. 1937. Pugillus fungorum nevadensium. Cavanillesia, 8: 133-137.

MAIRE, R. 1943. Fungorum nevadensium. pugillus alter autore. An J. Bot. Madrid, 3: 51-52.

MALENCON, G. 1968. Contribution à la flore mycologique de l'Andalousie Collect. Bot., 7: 707-725.

MALENCON, G. \& BERTAULT, R. 1970. Champignons superieurs du Maroc. I. Rabat.

MALENÇON, G. \& BERTUAUlT, R. 1971. Champignons de la Peninsule Iberique. Explorations entre le Midi valencien et le Montseny. Acta Pbytotax. Barcinonensia, 8: 1-94.

MALENÇON, G. \& BERTAULT, R. 1972. Champignons de la Peninsule Iberique. Les Iles Baleares. Acta Phytotax. Barcinonensia, 11: 1-64.

MALENÇON, G. \& BERTAULT, R. 1975. Champignons superieurs du Maroc. II. Rabat.

MALENÇON, G. \& BERTAULT, R. 1976. Champignons de la Peninsule Iberique. Catalogne, Aragon, Andalousie. Acta Pbyt. Barcinonensia, 19: 1-67.

MORENO, G. 1976. Contribución al estudio micológico de Andalucía. Acta Bot. Malacitana, 2: 5-20.

MUÑOZ MEDINA, J. M. \& SERRANO SANCHEZ, A. 1947. Notas micológicas para la flora andaluza. Bol. R. Soc. Esp. Hist. Nat., 45: 511-516.

RIVAS-MARTINEZ, S. \& LOSA QUINTANA, J. M. 1969. Comportement sociologique des champignons des dunes littorales du fleuve Llobregat (Barcelona). Bull.' Soc. Mycol. de France, 85: 235-244.

TORRE, M. de la. 1974. Estudio sistemático, ecológico y corológico de Ascomycetes españoles. Tesis Doctoral, Universidad de Madrid (pendiente de publicar).

UNAMUNO, L. 1941. Enumeración y distribución geográfica de los Ascomycetes de la Península Ibérica y de las Islas Baleares. R. Ac. Cien. Ex. Fis. y Nat. Madrid. 403 pp. 